\title{
Evidence that the fully assembled capsid of Leishmania RNA virus 1-4 possesses catalytically active endoribonuclease activity
}

\author{
Young Tae Ro ${ }^{1,4}$, Eun Ju Kim ${ }^{1}$ \\ Hyun II Lee ${ }^{1}$, Margarita Saiz ${ }^{2, *}$ \\ Ricardo Carrion $\mathrm{Jr}^{3}$ and Jean L. Patterson ${ }^{3}$ \\ ${ }^{1}$ Department of Biochemistry \\ College of Medicine, Konkuk University \\ Chungju 380-701, Korea \\ ${ }^{2}$ Department of Microbiology and Molecular Genetics \\ Harvard Medical School, Boston, MA 02115, USA \\ ${ }^{3}$ Department of Virology and Immunology \\ Southwest Foundation for Biomedical Research \\ San Antonio, Tx 78245-0549, USA \\ ${ }^{4}$ Corresponding author. Tel, 82-43-840-3739; \\ Fax, 82-43-851-3944; E-mail, ytaero@kku.ac.kr \\ *Present address: CISA-INIA, 28130 Valdeolmos, Madrid, Spain.
}

Accepted 7 February 2004

Abbreviations: LRV, Leishmania RNA virus; Sf9, Spodoptera frugiperda 9; VLPs, virus-like particles

\begin{abstract}
In this study, Leishmania RNA virus 1-4 (LRV1-4) particles purified from host Leishmania guyanensis promastigotes were examined for capsid endoribonuclease. Temperature optimum for the endoribonulease activity was found to be at $37^{\circ} \mathrm{C}$ to $42^{\circ} \mathrm{C}$ and the activity was specifically inhibited by the aminoglycoside antibiotics, neomycin, kanamycin, and hygromycin and by $100 \mathrm{mM}$ levels of $\mathrm{NaCl}$ or $\mathrm{KCl}$. To determine the catalytic domain of the capsid endoribonuclease activity, three point-mutation at cysteine residues at C47S (P1), C128/ 133S (P2), and C194R (P3) were prepared and each gene was constructed into baculoviruses and expressed in Sf9 insect cells. LRV1-4 capsid $N$ terminus (N2 and N3) and C-terminus (C1 and C2) deletion mutants (Cadd et al., 1994) were also examined by in vitro RNA cleavage assay. The results showed that the capsid mutants; $C 1, C 2$, N3, P1, and P2 were capable of forming proper virus-like particles (VLPs) and they all possessed the specific endoribonuclease activity. However, two assembly-defective capsid mutants, N2 (N-
\end{abstract}

terminus 24-amino acids deletion) and P3 mutants, did not retain the specific endoribonuclease activity. Taken together, the results suggest that at least 24 amino acids from the $\mathrm{N}$-terminal region and $\mathrm{C} 194$ residue in LRV1-4 capsid protein are functionally important for LRV1-4 viral assembly and the capsid endoribonuclease activity may be dependent upon the properly assembled LRV1-4 virus particles.

Keywords: Endoribonuclease; Leishmania virus; mutational analysis; RNA cleavage; viral assembly

\section{Introduction}

Members of the genus Leishmania are protozoan parasites and the causative agent of leishmaniasis in humans. Since the first double-stranded RNA (dsRNA) virus was discovered in Leishmania sp. in 1988 (Tarr et al., 1988; Widmer et al., 1989), similar viruses have been found in at least 13 cultured strains of Leishmania. These viruses are now designated as the genus Leishmania RNA virus (LRV) and classified as a member of the Totiviridae family of viruses. The complete sequences and molecular organizations have been reported for two New World isolates, LRV1-1 and LRV1-4, and one Old World isolate, LRV2-1 (Stuart et al., 1992; Scheffter et al., 1994; 1995, respectively). LRV1-4 genome consisting of a 5.3-kbp dsRNA possesses two large overlapping open reading frames (ORF2 and ORF3) constituting 90\% of the viral genome (Scheffter et al., 1994). When the ORF2 encoding 742 amino acids was expressed in a baculovirus expression system, the expressed protein self-assembled into virus-like particles (VLPs) of identical morphology to native virions (Cadd and Patterson, 1994), indicating that ORF2 does encode the capsid protein. To delineate the essential domains necessary for the viral assembly, several LRV1-4 capsid deletion mutants were generated by using the baculovirus expression system (Cadd et al., 1994). The two carboxyl (C)-terminal deletion mutants, C1 and $\mathrm{C} 2$, missing the last 115 and 24 amino acids, respectively, retained the ability to assemble, as determined by sucrose gradient sedimentation and electron microscopy (EM) analysis. Also, N3 mutant, which was missing only six amino acid from amino 
(N)-terminus of LRV1-4 capsid, was still fully competent for assembly, as determined by sedimentation and EM analysis. However, N2 mutant, missing only 24 amino acids from $\mathrm{N}$-terminus, was not able to assemble efficiently, suggesting that this $\mathrm{N}$-terminal region is essential for the viral assembly (Cadd et al., 1994).

In 1994, Chung et al. reported that a short RNA transcript in addition to genomic-length RNA transcript was generated by LRV virions in an in vitro polymerase assay. The short transcript is a singlestranded RNA (ss-RNA), corresponding to the $5^{\prime}$ end of positive-sense viral RNA genome. A putative cleavage site, conserved in LRV1-1 and 1-4 genomes, was mapped by primer extension analysis at nucleotide 320 of the viral genome and the endoribonuclease activity responsible for the cleavage event was proteinaceous in nature and was only associated with intact viral particles (MacBeth and Patterson, 1995a). In an in vitro RNA cleavage assay, baculovirus-expressed LRV1-4 capsid protein possessed an endoribonuclease activity identical to that of native virions, surprisingly showing that the viral capsid protein is the endoribonuclease responsible for generating the short RNA transcript (MacBeth and Patterson, 1995b). Further analysis showed that determinants necessary for the cleavage are encoded in viral nucleotide 249 to 342 , and that a stem-loop structure in this region stabilized by divalent metal ions $\left(\mathrm{Mg}^{2+}\right.$ or $\mathrm{Ca}^{2+}$ ) is essential for accurate RNA cleavage (Ro and Patterson, 2000).

The identification of a specific endoribonuclease activity of the LRV capsid protein which functions on its own RNA genome was unprecedented for viral capsid because the functions of viral capsid are assumed to be mainly for the maintenance of virus structure and protection of viral genomes. Also, when the amino acid sequence of LRV1-4 capsid protein (EMBL/GenBank U01899) was analyzed by using PROSITE database (Falquet et al., 2002), the capsid protein did not bear any known RNase motifs. Therefore, even though extensive evidences have been reported that the cleavage activity is solely dependent on LRV capsid protein (MacBeth and Patterson, 1995a; b; MacBeth et al., 1997; Ro and Patterson, 2000), further detailed analyses are required to determine whether LRV1-4 capsid protein truly possesses the enzymatic properties of the endoribonuclease activity responsible for the single-site cleavage in the 5'-untranslated region (UTR) of the genome. In this study, we report some biochemical properties of LRV1-4 capsid protein as an endoribonuclease. We also characterize possible catalytically functional domains for the RNA cleavage using baculovirusexpressed LRV1-4 capsid deletion mutants previously constructed (Cadd et al., 1994) and three pointmutated LRV1-4 capsid mutants at cysteine residues.

\section{Materials and Methods}

\section{Parasite strain and cell culture}

Leishmania guyanensis M4147 (MHOM/BR/75/M4147) was grown at $23^{\circ} \mathrm{C}$ in $\mathrm{M} 199$ semidefined medium (Gibco Laboratories) supplemented with $5 \%$ fresh, filter-sterilized human urine (Armstrong and Patterson, 1994).

\section{Construction of recombinant baculoviruses}

The three point mutants of LRV1-4 capsid carrying substitutions in the $\mathrm{N}$-terminal half (P1, P2, and $\mathrm{P} 3$ ) were generated by using PCR-direct mutagenesis as previously described (Oh et al., 2002) and the primers listed in Table 1. Briefly, for the construction of mutant $\mathrm{P} 1$ which contains a substitution of Cys-47 residue to Ser (C47S), a primer (P1-L) containing a mismatch at $n t 140$ ( $G$ to $C)$ and polyhedrin reverse sequencing primer (Ph-Rev, Invitrogen) were used to prime a DNA fragment synthesis from pTCB2 (Cadd and Patterson, 1994) as a template. Similarly, a primer (P1-R) containing a mismatch at nt 140 (C to $G)$ and polyhedrin forward sequencing primer (Ph-For, Invitrogen) were also used to prime a DNA fragment synthesis from $p T C B 2$. Two PCR products were gelpurified, combined, and heat-denatured. Then, the PhFor and Ph-Rev primers were added in the mixture and the second PCR was performed. Mutagenesis of residues $C 194 R$ was performed in a similar way using each set of primers listed in Table 1 to generate mutant P3. Mutant P2 carrying double point mutations (C128S and C133S) was generated using a set of primers (P2-L/P2-R) containing two mismatches in position nt 383 and 398 . The secondary PCR products were gel-purified and digested with Pstl and the

Table 1. Oligonucleotides used for the construction of capsid point mutants.

\begin{tabular}{|c|c|}
\hline Name & Sequence $^{a} \quad\left(5^{\prime} \longrightarrow 3^{\prime}\right)$ \\
\hline P1-L & TTGAACTATTCETTCAACCG \\
\hline P1-R & CGGTTGAAGAATAGTTCAA \\
\hline P2-L & GTGCATㅁTATCGCACATTATTㅡㄷATGAC \\
\hline P2-R & GTCATG $\underline{G A A T A A T G T G C G A T A G A T G C A C}$ \\
\hline P3-L & GGTGCGCTEGGTGACGCTAG \\
\hline P3-R & CTAGCGTCACGAGCGCACC \\
\hline Ph-For & AAATGATAACCATCTCGC \\
\hline Ph-Rev & GTCCAAGTTTCCCTG \\
\hline
\end{tabular}

\footnotetext{
a, Mutated bases are highlighted with boldface and underline.
} 


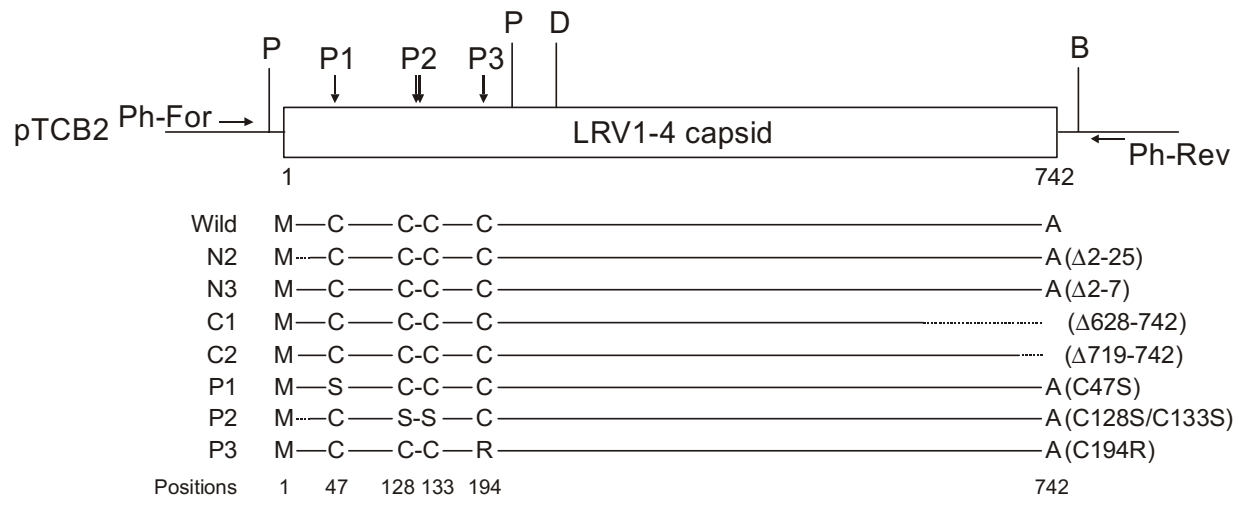

Figure 1. Schematic representation of the LRV1-4 capsid in PTCB2 baculovirus expression vector (Cadd and Patterson, 1994) and an illustrated map of the capsid mutant constructs. The sites of point mutations were indicated by arrows. Amino acid positions are based on LRV1-4 capsid protein (EMBL/ GenBank U01899). Abbreviations of restriction enzymes: B (BamHI), $\mathrm{D}$ (DrallI), and $\mathrm{P}$ (Pstl). resulting $678 \mathrm{nt}$ fragments were gel-purified and individually ligated into $p T C B 2$ digested with Pstl. A schematic representation of the partial pTCB2 map, including the locations of the point mutated regions and restriction endonuclease sites used for capsid mutants construction is shown in Figure 1. All the specific mutations from the resulting vectors were confirmed by DNA sequencing.

The generated transfer vectors (each $3 \mu \mathrm{g}$ ) were transfected into Spodoptera frugiperda 9 (Sf9) cells along with $1 \mu \mathrm{g}$ of linear baculovirus DNA, and recombinant plaques were identified and purified, according to the manufacturer's instructions (Linear AcMNPV DNA Transfection Module, Invitrogen). Expression of mutant capsid proteins was checked by Western blot analysis with LRV1-4 capsid antiserum (Cadd et al., 1993), as previously described (Ro et al., 1997a).

\section{Sucrose gradient sedimentation of LRV1-4 native virus and baculovirus-expressed VLPs}

Native LRV1-4 virions from M4147 promastigotes were purified by a 10 to $40 \%$ sucrose gradient as previously described (Cadd and Patterson, 1994). VLPs from wild-type or mutant capsids were generated by infecting a $60-\mathrm{mm}$ diameter tissue culture dish containing $2 \times 10^{6}$ Sf9 cells with the recombinant baculoviruses at a multiplicity of infection (MOI) of 10 . The infected cells were harvested at day 3 postinfection by scraping the cells from the dish in 0.5 $\mathrm{ml}$ of lysis solution (50 mM Trizma base [pH 8.0], 150 $\mathrm{mM} \mathrm{NaCl}, 2 \%$ Nonidet P-40), loaded onto an 11-ml, 10 to $40 \%$ sucrose gradient in $\mathrm{HCN}$ buffer $(50 \mathrm{mM}$ $\mathrm{N}$-2-hydroxyethylpiperazine-N'-2-ethanesulfonic acid [HEPES; $\mathrm{pH} 7.4$ ], $5 \mathrm{mM} \mathrm{CaCl}_{2}, 150 \mathrm{mM} \mathrm{NaCl}$ ), and centrifuged at $36,000 \mathrm{rpm}$ in a Beckman SW41 rotor for $135 \mathrm{~min}$ at $4^{\circ} \mathrm{C}$. Sucrose gradients were collected in 1-ml fractions. Additionally, wild-type LRV1-4 capsid and other LRV1-4 capsid mutants [two N-terminus deletion mutants (N2 and $\mathrm{N} 3$ ) and two C-terminus deletion mutants ( $\mathrm{C} 1$ and $\mathrm{C} 2$ )] previously constructed
(Cadd et al., 1994) were similarly expressed in Sf9 cells and included in the analysis. Both native and recombinant VLPs were analyzed by Western blot analysis with LRV1-4 capsid antiserum, and used in RNA cleavage assay.

\section{Substrate RNA synthesis for RNA cleavage assay}

Plasmid DNA template ( $p B S K 5$ '249-342) containing LRV1-4 5' 249-342 region described previously (Ro and Patterson, 2000) was linearized by digestion with EcoRI, and in vitro transcription was accomplished by T7 RNA polymerase (Promega) according to the manufacturer's protocol. Briefly, transcription reaction mixture was incubated $37^{\circ} \mathrm{C}$ for $2 \mathrm{~h}$, and template DNA was removed by treating with $R Q 1$ DNase (Promega) for $15 \mathrm{~min}$ at $37^{\circ} \mathrm{C}$. The RNA product was treated with calf intestine phosphatase [New England Biolabs (NEB)], extracted twice with phenol-chloroform, and precipitated with ethanol. The dephosphorylated RNA was $5^{\prime}$ end labeled with T4 polynucleotide kinase (NEB) and $\left[\gamma^{32} \mathrm{P}\right]$ ATP (Dupont-NEN), and the labeled RNA was purified as previously described (MacBeth et al., 1997), and used in RNA cleavage assay.

\section{In vitro RNA cleavage assay}

The cleavage activity was assayed in $20-\mu$ l reaction mixture containing the labeled substrate RNA $(100,000$ cpm), sucrose-purified viral particles at $20 \%$ volume, and $20 \mathrm{U}$ of RNasin (Promega), as described previously (MacBeth and Patterson, 1995a). Incubation was at $37^{\circ} \mathrm{C}$ for $40 \mathrm{~min}$, and a portion of each reaction mixture was mixed with formaldehyde loading dye and heat denatured at $90^{\circ} \mathrm{C}$ for $2 \mathrm{~min}$. The reaction products were resolved on a denaturing $8 \%$ polyacrylamide-8.3 M urea gel and visualized by autoradiography. The labeled substrate RNA was also digested with RNase $T_{1}$ (Rosenstein and Been, 1991) and used for RNA size marker of the cleavage product. For biochemical analysis of RNA cleavage activity, various conditions were applied as described 
in each figure legend.

\section{Results}

\section{Biochemical properties of LRV1-4 capsid endoribonuclease}

Earlier report showed that LRV1-4 capsid protein cleaved the minimum RNA substrate 5'249-342 at the nucleotide 320 and near the nucleotide 276 region that contains the essential stem-loop structure for site-specific RNA cleavage, and that LRV1-4 capsidspecific antiserum blocked the cleavage at the nucleotide 320 , but not around the nucleotide 276 region (Ro and Patterson, 2000). To further delineate the specificity of LRV1-4 capsid endoribonuclease activity, native LRV1-4 virus particles were pre-incubated with

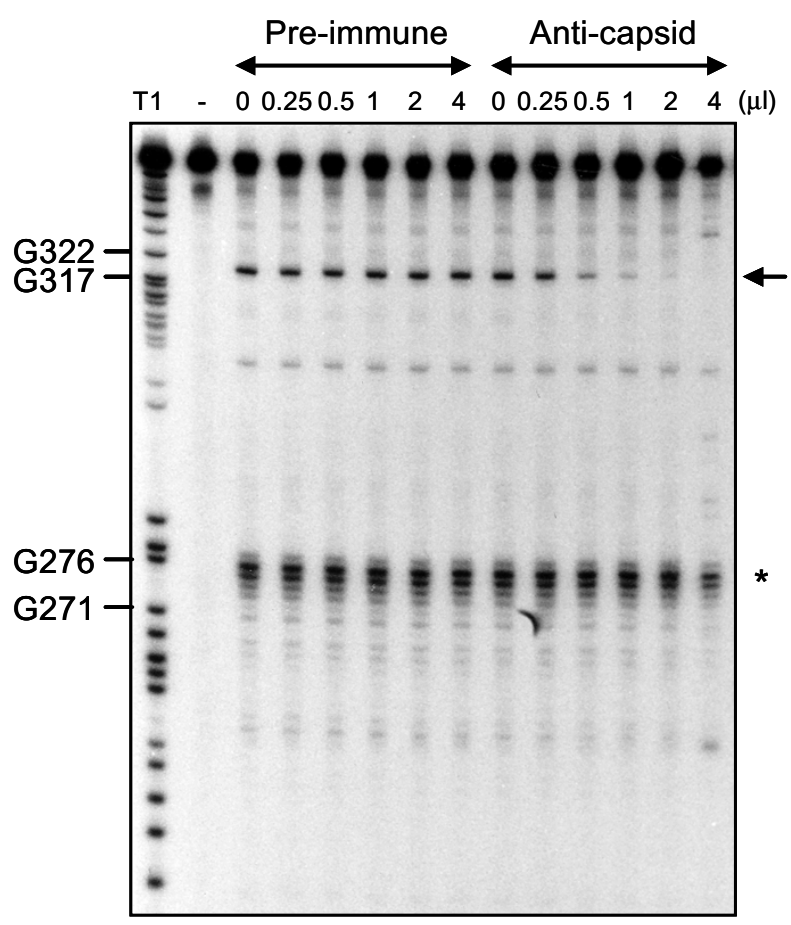

Figure 2. Specific inhibition of LRV1-4 capsid endoribonuclease activity by LRV1-4 capsid-specific antiserum. A $3-\mu$ I aliquot of a sucrose gradient fraction containing native LRV1-4 viral particles (6 $\mu \mathrm{g}$ of total protein) was preincubated with increasing amounts (from $0.25 \mu \mathrm{l}$ to $4 \mu \mathrm{l}$ of each one-tenth diluted antisera) of preimmune serum (pre-immune) or LRV1-4 capsid protein-specific antiserum (anti-capsid). After 10 min of incubation at room temperature, the RNA cleavage activity was assayed with the RNA substrate 5'249-342 for $40 \mathrm{~min}$ as described in Materials and Methods. Lane (-) indicates a negative control without LRV1-4 viral particles. Arrow and asterisk indicate the $320 \mathrm{nt}$ cleavage product and the cleavage products near the nucleotide 276 region, respectively. RNA molecular weight marker (T1) was generated by partial digestion of the RNA substrate with RNase T1 $\left(5 \times 10^{-3} \mathrm{U} / \mu \mathrm{l}\right.$, final concentration) and molecular sizes were indicated as nucleotide positions in the substrate RNA5'249-342. increasing titers of pre-immune or LRV1-4 capsidspecific antiserum for $10 \mathrm{~min}$ and the RNA cleavage activity was assayed with the minimum RNA substrate 5'249-342 as described in Materials and Methods. The results showed that increasing titers of preimmune serum did not block either the cleavage at the nucleotide 320 (arrow) or the cleavage at the nucleotide 276 region (asterisk) (Figure 2), as previously described (Ro and Patterson, 2000). However, increasing titers of LRV1-4 capsid-specific antiserum specifically abolished the cleavage only at the nucleotide 320 , but not at the nucleotide 276 region, showing that the specific-cleavage at the nucleotide 320 is truly mediated by LRV1-4 capsid protein.

When the RNA cleavage activity of native LRV1-4 virus particles was assayed at various temperatures for $40 \mathrm{~min}$, the optimum temperature for the specific cleavage activity was at $37^{\circ} \mathrm{C}$ to $42^{\circ} \mathrm{C}$ (Figure $3 \mathrm{~A}$ ). When the RNA cleavage assay was conducted for 40 min at higher temperature over $50^{\circ} \mathrm{C}$, most of the labeled RNA substrate was fragmented and the cleavage products at the nucleotide 276 region were proportionally increased (Figure $3 \mathrm{~A}$ ), showing that the prolonged incubation at high temperature over $50^{\circ} \mathrm{C}$ may cause the RNA degradation and the products at the nucleotide 276 region may be by-products of the

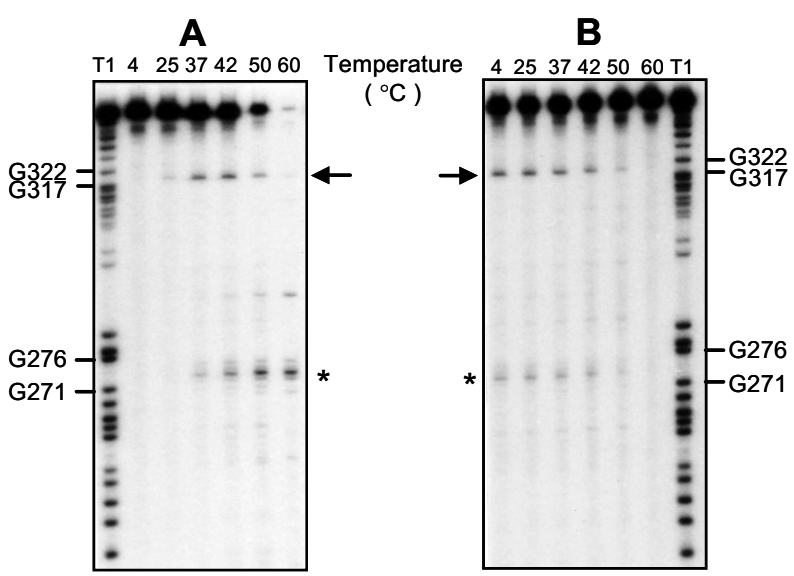

Figure 3. Effects of temperature on LRV1-4 capsid endoribonuclease activity. (A) Optimal temperature for endoribonuclease activity. Native LRV1-4 virus particles purified by sucrose gradient fractionation were subjected to an in vitro RNA cleavage assay for 40 min as described in Materials and Methods at the indicated temperatures. (B) Thermal stability of LRV1-4 capsid endoribonuclease. Equal amounts $(6 \mu \mathrm{g}$ of total protein) of the purified native LRV1-4 virus particles were individually incubated at the indicated temperatures for $10 \mathrm{~min}$ prior to an in vitro RNA cleavage assay and then the residual RNA cleavage activity was assayed for $40 \mathrm{~min}$. Arrow and asterisk indicate the $320 \mathrm{nt}$ cleavage product and the cleavage products near the nucleotide 276 region, respectively. RNA molecular weight marker (T1) was generated by partial digestion of the RNA substrate with RNase T1 $\left(5 \times 10^{-3} \mathrm{U} / \mu \mathrm{l}\right.$, final concentration) and molecular sizes were indicated as nucleotide positions in the substrate RNA5'249-342. 


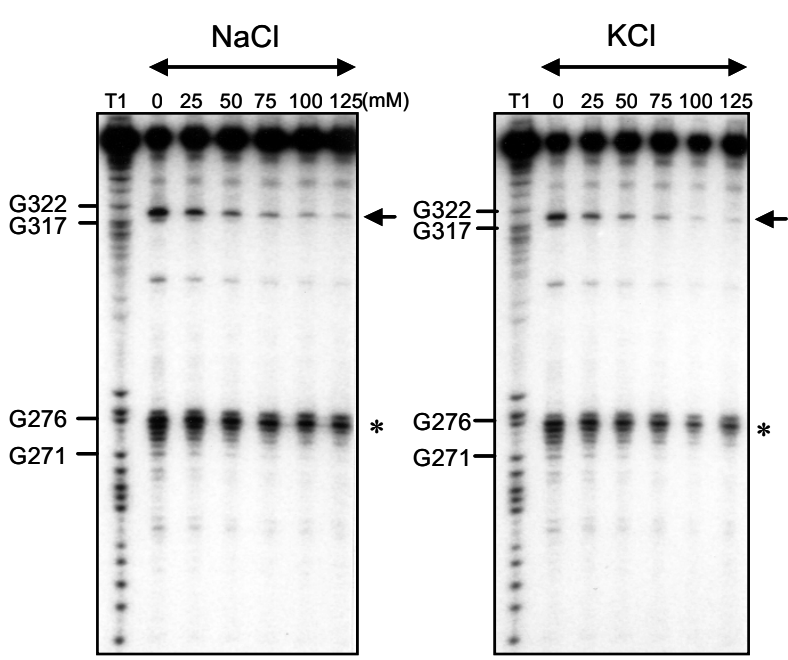

Figure 4. Inhibitory effects of $\mathrm{NaCl}$ and $\mathrm{KCl}$ on LRV1-4 capsid endoribonuclease activity. Equal amounts ( $6 \mu \mathrm{g}$ of total protein) of the purified native LRV1-4 virus particles were subjected to an in vitro RNA cleavage assay at the different concentration of salts indicated. Arrow and asterisk indicate the $320 \mathrm{nt}$ cleavage product and the cleavage products near the nucleotide 276 region, respectively. RNA molecular weight marker (T1) was generated by partial digestion of the RNA substrate with RNase T1 $\left(5 \times 10^{-3} \mathrm{U} / \mathrm{ml}\right.$, final concentration) and molecular sizes were indicated as nucleotide positions in the substrate RNA5'249-342.

non-specific viral particle mediated degradation. RNA substrate alone was unaffected during the incubation without virus particle fraction at $60^{\circ} \mathrm{C}$ for $40 \mathrm{~min}$ (data not shown). Heat sensitivity of the viral particle fraction was examined by pre-treating the particle fraction $10 \mathrm{~min}$ at various temperatures and the enzyme activity was carried out by in vitro RNA cleavage assay. The endoribonuclease activity responsible for the specific cleavage was mostly inactivated at $50^{\circ} \mathrm{C}$ (Figure 3B). The endoribonuclease activity was not effected by $\mathrm{pH}$ between 6.0 through 9.0 (data not shown). Salts that are known to influence the nucleotide dissociation were tested on the capsid-dependent RNA cleavage. Addition of increasing amounts of $\mathrm{NaCl}$ or $\mathrm{KCl}$ to RNA cleavage reaction mixtures resulted in a dose-dependent inhibition of the specificRNA cleavage activity and almost completely at 100 $\mathrm{mM}$ salt treatment (Figure 4). In contrast, RNase T1 activity was not affected by salt even at over $150 \mathrm{mM}$ concentration. To test whether the loss of the endoribonuclease activity by salt is caused by virusparticle dissociation in high salt conditions, the virus particles pre-treated with $150 \mathrm{mM}$ of $\mathrm{NaCl}$ or $\mathrm{KCl}$ for $40 \mathrm{~min}$ were mixed with SDS-PAGE loading buffer without $\beta$-mercaptoethanol and subjected to a SDSPAGE and Western blot analysis with LRV1-4 capsid specific antiserum. The results showed that no dissociated capsid protein from virus particle was detected (data not shown), indicating that the salt concentration we tested has no effects for LRV1-4 virus particle dissociation.

Previously, we reported that hygromycin B, an aminocyclitol antibiotic, mediates elimination of LRV from its persistently infected parasite host (Ro et al., 1997b). To test a possible association of such antibiotic reaction of hygromycin $B$ with the endoribonuclease activity, increasing doses of the antibiotic were added to the RNA cleavage reaction medium. The result showed a dose-dependent inhibition of specific RNA cleavage activity at the nucleotide 320 whereas the non-specific cleavage activity was unaffected even at $40 \mathrm{mM}$ hygromycin B (Figrue 5). Neomycin and kanamycin also showed the strong inhibition of the specific RNA cleavage inhibition even at $20 \mu \mathrm{M}$ and $0.9 \mathrm{mM}$, respectively, but phleomycin and ampicillin did not inhibit the RNA cleavage activity at 1.2 and $1.0 \mathrm{mM}$, respectively (Figrue 5). When ampicillin was added in the RNA cleavage mixture at $2 \mathrm{mM}$ concentration, the cleavage activity seemed to be slightly enhanced (Figrue 5). However, a higher dose of ampicillin $(10 \mathrm{mM})$ treatment did not show any further enhancement or inhibition of the RNA cleavage activity (data not shown). We could not examine the phleomycin effect over $1.2 \mathrm{mM}$ concentration on the RNA cleavage activity, due to mobility retardations of the substrate RNA as shown in Figrue 5. The recombinant wild type LRV1-4 capsid protein expressed in sf9 cells showed a similar pattern of inhibition as the native virus particles did.

\section{Expression of recombinant viral capsid proteins and Western blot analysis}

Assembled virus particles from either wild-type or mutant LRV1-4 capsid proteins, generated by baculovirus expression were reported to be sedimented with a peak between fraction 4 through 6 (middle fractions) in a sucrose gradient (Cadd et al., 1994). Unassembled proteins, however, remained at the top of the gradient or in the pellet. Due to this correlation of a specific sedimentation pattern through a sucrose gradient with the assembly into VLPs, the point-mutated LRV1-4 capsid proteins prepared in this study were analyzed for their sedimentation properties by sucrose gradients centrifugation, as an indication marker of their levels of assembly.

The wild-type capsid and capsid mutants (wild, C1, $\mathrm{C} 2$, N2, and N3) were constructed as previously described (Cadd and Patterson, 1994; Cadd et al., 1994) and P1, P2, and P3 were individually expressed in sfg insect cells and their capsid protein expression levels were examined by Coomassie blue staining and Western blot analysis with antiserum against LRV1-4 capsid protein. When equal amounts (40 $\mu \mathrm{g}$ of total proteins each) of cell lysates from 

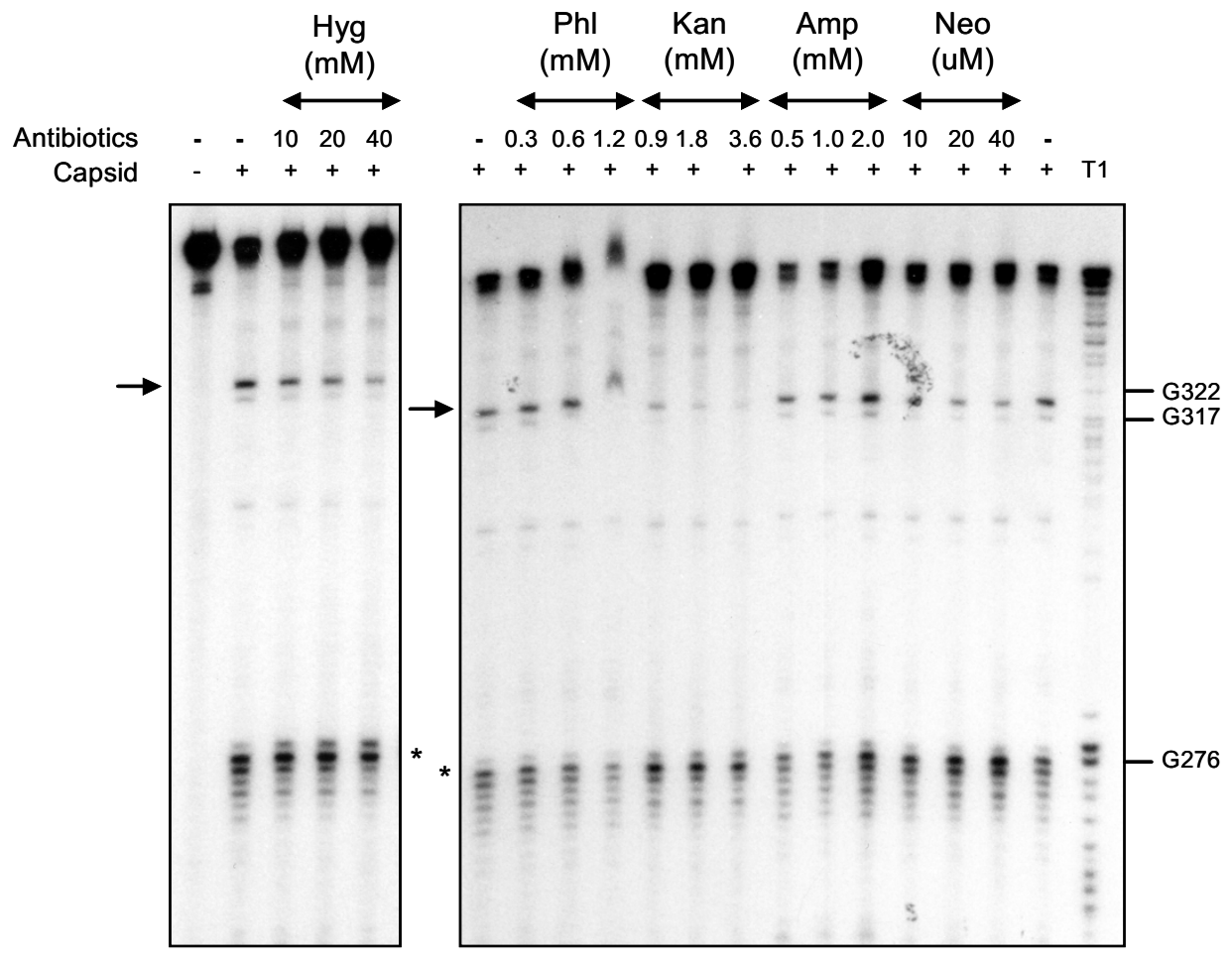

Figure 5. Effect of antibiotics on LRV1-4 capsid endoribonuclease activity. Equal amounts $(6 \mu \mathrm{g}$ of total protein) of the purified native LRV1-4 virus particles were subjected to an in vitro RNA cleavage assay at the concentration of antibiotics indicated. Arrow and asterisk indicate the $320 \mathrm{nt}$ cleavage product and the cleavage products near the nucleotide 276 region, respectively. RNA molecular weight marker (T1) was generated by partial digestion of the RNA substrate with RNase T1 $\left(5 \times 10^{-3}\right.$ $\mathrm{U} / \mathrm{ml}$, final concentration) and molecular sizes were indicated as nucleotide positions in the substrate RNA5'249-342. Abbreviations: Hyg (hygromycin), Phl (phleomycin), Kan (kanamycin), Amp (ampicillin), and Neo (neomycin).

different recombinant mutants were analyzed by Coomassie blue staining after 10\% SDS-PAGE, the recombinant capsid proteins from the baculovirusexpressed deletion capsid mutants (N3, C1, and C2) were expressed as similar levels of the wild-type capsid protein and the recombinant $\mathrm{N} 2$ capsid protein was expressed at a much lower level than the wild-type capsid protein (arrow in Figure 6A), closely similar to the results previously shown by Cadd et al. (1994). Among the newly prepared point-mutated capsid proteins; $P 1, P 2$, and $P 3$, generated in this study, the recombinant $\mathrm{P} 1$ and $\mathrm{P} 2$ capsid proteins were highly expressed as similar levels to the wildtype capsid protein, but the expression level of the P3 mutant was much lower than the wild-type capsid protein (Figure 6A). To investigate the viral assembly of the recombinant capsid mutants by their sedimentation properties in the sucrose gradient, the prepared cell lysates were individually loaded onto a $5.5 \mathrm{ml}$ column with 10 to $40 \%$ sucrose gradient, centrifuged, and fractionated into $1-\mathrm{ml}$ aliquote. When $10 \mu$ aliquotes of each fraction were analyzed by Western blot analysis after 10\% SDS-PAGE, the major proteins recognized by LRV1-4 antiserum appeared as the expected sizes, as predicted from mutants sequence information, and the capsid protein distributions of previously constructed capsid mutants $(C 1, C 2, N 2$, and N3) and wild-type capsid were similar to the results previously reported (Cadd et al., 1994) and they peaked at the fraction 5 (Figure 6B).
Also, the wild-type capsid and recombinant capsid proteins appeared to be a doublet with a fastermigrating capsid species that is previously described as a putative cleavage product of the viral capsid (Cadd et al., 1993). The sedimentation profiles of the capsid proteins prepared from the newly constructed point-mutated capsid proteins; $\mathrm{P} 1$ and $\mathrm{P} 2$, also appeared similarly to that of the wild-type capsid in that the majority of the recombinant capsid proteins peaked at the fraction 5 (Figure 6B). However, the expression level and sedimentation profile of the recombinant $\mathrm{P} 3$ mutants appeared to be similar to those of the assembly-defective N2 mutant (Cadd et al., 1994). The majority of the immuno-cross reacting protein produced from $\mathrm{N} 2$ mutants was found in the pellet of the sucrose gradient (data not shown), as previously reported (Cadd et al., 1994), and several faster migrating proteins than the wild-type capsid protein were also recognized by the polyclonal sera (Figure $6 \mathrm{~B}$ ), indicating that the $\mathrm{N} 2$ recombinant capsid is assembly-defective. Similarly, the recombinant P3 capsid mutants sedimented primarily in the pellet and partially at the middle of gradient, peaked at the fraction 5 (Figure 6B), showing that they are partially or improperly assembled into VLPS.

For overall comparison of all the recombinant capsid proteins along with the wild-type capsid protein, the fraction 5 of each preparation was analyzed because all the wild-type and recombinant particles were most abundant in this fraction. Twenty microliter 


\section{A}

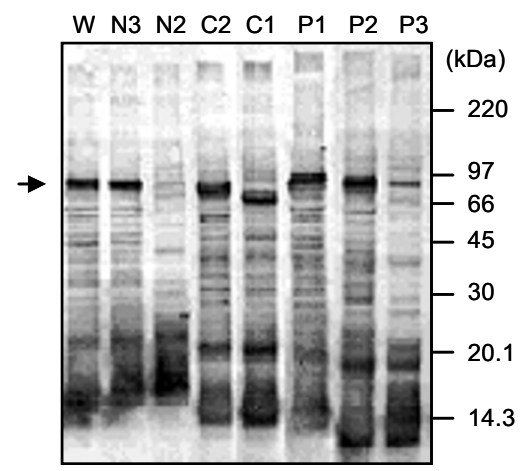

B
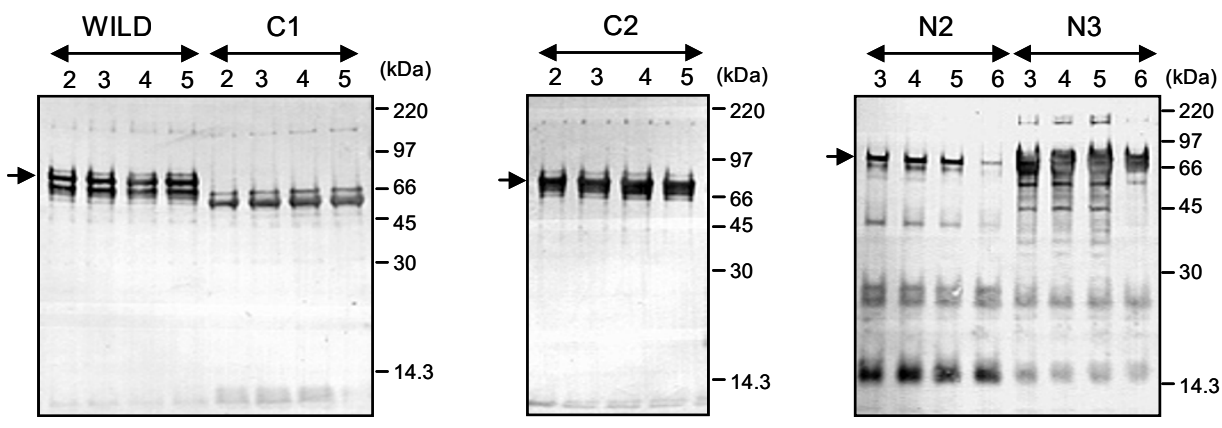

Figure 6. Comparison of the expressions of LRV1-4 wild type and recombinant mutant capsid proteins. (A) Coomassie blue staining. Equal amounts $(40 \mu \mathrm{g}$ of total proteins each) of cell lysates from wild-type (W) and capsid mutants described in Table 2 were analyzed by Coomassie blue staining after a $10 \%$ SDS-PAGE. The capsid proteins predicted by the expected sizes are indicated as an arrow. (B) Western blot analysis. The cell lysates from different recombinant capsid proteins were individually loaded onto a 10 to $40 \%$ sucrose velocity gradient $(5.5$ $\mathrm{ml}$ each), centrifuged, and collected into $1-\mathrm{ml}$ fractions and numbered from the bottom of the gradient. Ten $\mu \mathrm{l}$ of each fractions indicated as numbers were analyzed by Western blot analysis with LRV1-4 capsid-specific antiserum after $10 \%$ SDS-PAGE. Capsid doublets are indicated as an arrow.

aliquots from each fraction five preparations separated in a 10\% SDS-PAGE and blotted with LRV1-4 capsid antiserum showed that the majority of the immunoreacting proteins appeared to be doublet and their levels were similar, except those of recombinant N2 and $\mathrm{P} 3$ capsid proteins that were relatively lower than others (Figure 7A). Interestingly, an $180-\mathrm{kDa}$ protein previously depicted as oligomer of capsid protein not dissociating under the denaturing gel conditions (Cadd and Patterson, 1994; Ro et al., 1997a) was detected only in $\mathrm{C} 1, \mathrm{C} 2, \mathrm{~N} 3, \mathrm{P} 1$, and $\mathrm{P} 2$ recombinant capsid fractions, similar as in the wild-type capsid fraction (Figure 7A). However, the 180-kDa capsid protein was not seen in the assembly-defective N2 and $\mathrm{P} 3$ capsid mutant fractions (Figure 7A). To test whether the lack of the capsid dimer in N2 and P3 mutant fractions may be due to the lower capsid concentration in these fractions, the 10-fold concentrated N2 and P3 mutant capsid fractions using Microcon YM-30 filter units (Amicon) were examined. The capsid dimer was not detected at all in both concentrates, even though a comparable amount of capsid proteins were visualized (data not shown), indicating that the $\mathrm{N} 2$ and $\mathrm{P} 3$ capsid proteins are defective of the capsid dimer formation and of proper viral assembly.

\section{RNA cleavage activity of the recombinant viral capsid proteins}

To investigate whether the recombinant capsid mutants described above possess the endoribonuclease 
activity, equal amounts of proteins (1 $\mu \mathrm{g}$ each) in the fraction 5 from each recombinant capsid fractions were subjected to the RNA cleavage assay. The result showed that only the recombinant capsid fractions; N3, C1, C2, P1, P2, and the wild-type recombinant capsid protein, which all appeared to be properly assembled into VLPs (Cadd et al., 1994),

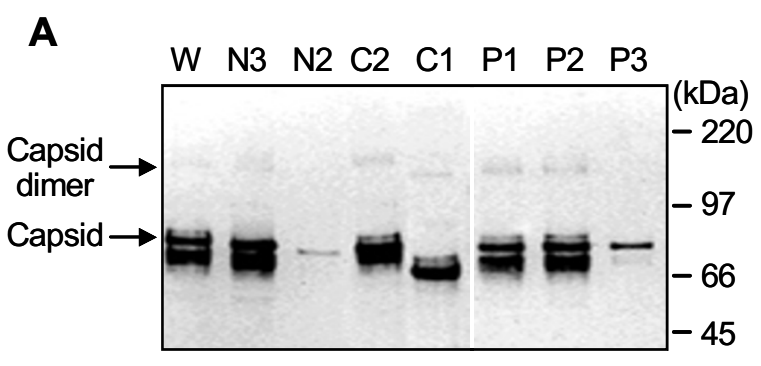

B

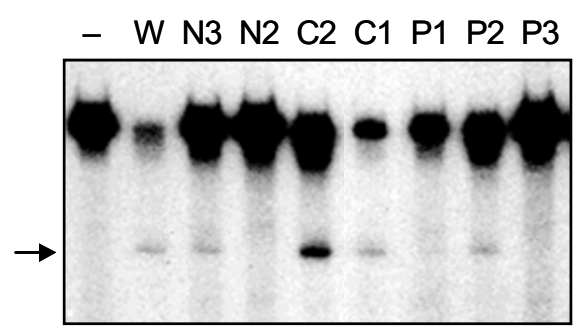

Figure 7. Western blot and in vitro RNA cleavage assay of LRV1-4 wild type and mutant capsid proteins. (A) Western blot analysis. Equal amounts $(20 \mu \mathrm{l}$ each) of each No. 5 fraction shown in Fig. 6B were subjected to a $10 \%$ SDS-PAGE and Western blot analysis was performed. Molecular sizes from Rainbow markers (Amersham Pharmacia) are indicated as kilodalton $(\mathrm{kDa})$ on the right. (B) Equal amounts (approximately $1 \mu \mathrm{g}$ each) of each No. 5 sucrose gradient fraction from wild-type (W) and capsid mutants were subjected to in vitro RNA cleavage assay, as described in "Materials and Methods". Arrows indicate the 320 nt cleavage product. cleaved the RNA substrate and specifically generated the $320 \mathrm{nt}$ cleavage product, even though the cleavage efficiency was relatively different (Figure 7B). However, two assembly-defective capsid mutants, N2 and P3, showed no RNA cleavage activity at all (Figure 7B), even at the 10 -fold elevated protein concentrates.

The results of virus assembly and RNA cleavage activity studies are summarized on Table 2 .

\section{Discussion}

In general, viral capsid proteins exclusively serve functions that ensure the successful propagation of viral genomes and protect viral genomes from nucleases in the intracellular and extracellular environments. Although LRV1-4 capsid protein was reported to possess an endoribonuclease activity for specific targeting on its own RNA (MacBeth and Patterson, 1995a and b; Ro and Paterson, 2000), the probable inclusion of host endoribonuclease(s) participating on the cleavage event of viral RNA can not be ruled out. In this study, native LRV1-4 virus particles was affirmatively demonstrated to cleave the minimum RNA substrate 5'249-342 selectively at the nucleotide 320 and the cleavage at the nucleotide 320 was specifically blocked by LRV1-4 capsid antiserum. In this in vitro RNA cleavage assay with native LRV1-4 virus particle fraction, another cleavage products resulting from cleavages at near the nucleotide 276 region are also appeared. The nature of this non-specific cleavage are totally different from that of the specific cleavage in that their cleavage rates were unaffected by LRV1-4 capsid-specific antiserum, salt, and aminoglycoside antibiotics treatments. The region around the nucleotide 276 is part of the stem-loop structure that

Table 2. Summary of RNA cleavage activity of LRV1-4 capsid mutants.

\begin{tabular}{|c|c|c|c|c|}
\hline Name & Description & Dimer formation ${ }^{a}$ & Assembly ${ }^{b}$ & 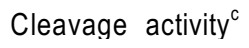 \\
\hline W & Wild-type capsid (1 742) & yes & + & + \\
\hline N2 & $\mathrm{N}$-terminus deletion (2 25) & no & - & - \\
\hline N3 & N-terminus deletion (2 7) & yes & + & + \\
\hline $\mathrm{C} 1$ & C-terminus deletion (628 742) & yes & + & + \\
\hline $\mathrm{C} 2$ & C-terminus deletion (719 742) & yes & + & + \\
\hline P1 & Single point mutation (C47S) & yes & + & + \\
\hline P2 & Double point mutations (C130S,C135S) & yes & + & + \\
\hline P3 & Single point mutation (C196R) & no & - & - \\
\hline
\end{tabular}

, Relative amount of cross-reacting protein which is present in fraction No. 5 of a 10 to $40 \%$ sucrose gradient, judged by Western blot analysis. ', Assembly was judged by Western blot (P1, P2, and P3) or/and electron microcopy analysis (W, N2, N3, C1, and C2) after sucrose gradient. ', Cleavage activity was determined by in vitro RNA cleavage assay. 
is known to be an essential component of the RNA cleavage reaction (Ro and Patterson, 2000) and renders single-stranded loop structure. At high temperature over $50^{\circ} \mathrm{C}$, the secondary structure of this region can easily retain a single-stranded nature that is more susceptible to cleavages by single-stranded specific endoribonuclease. The results that the added RNA substrate in the RNA cleavage assay condition at $60^{\circ} \mathrm{C}$ was almost disappeared, possibly resulting in the enhancement of the cleavage products at the nucleotide 276 region, suggested a possibility that single-stranded specific endoribonuclease(s) was contaminated in virus particle preparation, not in the substrate RNA preparation because the substrate RNA alone without virus particles fraction was not degraded at all during the prolong incubation at $60^{\circ} \mathrm{C}$ for $40 \mathrm{~min}$. However, it is most unlikely that the virus particles fraction we prepared has contained ribonucleases, resistant to heat-denaturation, in that the heat-treated virus fraction at $60^{\circ} \mathrm{C}$ for $10 \mathrm{~min}$ before in vitro RNA cleavage assay lost both the specific and non-specific cleavage activities at the nucleotide 320 and near the nucleotide 276 region, respectively. Therefore, this non-specific cleavage near the nucleotide 276 region may be also mediated by LRV1-4 capsid endoribonuclease activity. Taken together, these results further illustrate that the site-specific cleavage at the nucleotide 320 is truly mediated by LRV1-4 capsid protein.

The LRV1-4 capsid endoribonuclease activity exhibited a narrow optimal temperature, heat-instability at over $50^{\circ} \mathrm{C}$, and $\mathrm{pH}$ optimum at $\mathrm{pH} 6$ to 9 . Furthermore, the endoribonuclease activity was strongly inhibited by $\mathrm{NaCl}$ or $\mathrm{KCl}$ at over $100 \mathrm{mM}$, similar to $E$. coli ribonuclease III that has been shown to have an optimal activity at low salt concentration, below 60 $\mathrm{mM}$ (Sun et al., 2001). The reduction of LRV1-4 capsid endoribonuclease activity in high salt concentration may be caused either by that high salts interfere the interactions between RNA substrate and LRV1-4 capsid and/or disrupt the stem-loop structure in the substrate RNA 5'249-342 which is known to be an essential component of the cleavage reaction (Ro and Patterson, 2000). Since the single-strand specific RNase T1 identically cleaved the substrate RNA 5'249-342 in increasing salt concentration even at $150 \mathrm{mM}$ of $\mathrm{NaCl}$ or $\mathrm{KCl}$, it is believed that high salt does not affect the RNA secondary structure in the stem-loop region, but instead they interfere with the interaction of the substrate RNA 5'249-342 and LRV1-4 capsid protein. The salt concentrations (over $100 \mathrm{mM}$ ) used in this study did not cause the dissociation of LRV1-4 virus particles. Therefore, we ruled out a possibility that high salt can disrupt LRV particles and the dissociated virus particles lose their endoribonuclease activity.
An interesting property of LRV1-4 capsid endoribonuclease activity is the specific inhibition by some antibiotics, such as kanamycin, neomycin, and hygromycin B. These antibiotics are members of aminoglycoside antibiotics that possess a linked ring system consisting of aminosugars and an aminocyclitol (Bakker, 1992). Neomycin and kanamycin have found clinical use as antibacterial agents, because of the ability to bind specifically to prokaryotic rRNA and inhibit bacterial protein synthesis (Spahn and Prescott, 1996). It has been known that neomycin B and its arginine derivatives are a potent inhibitor of ribozymes such as bacterial and eukaryotic RNase $P$ (Mikkelsen et al., 1999; Tekos et al., 2000) and the self-splicing group I intron (von Ahsen et al., 1991) likely due to its ability to interfere with the binding of divalent metal ions essential for RNA catalysis (Eubank et al., 2002). In addition, the role of $\mathrm{Mg}^{2+}$ ions has been shown to affect the formation and stability of three-dimensional structures in many other types of RNA, including tRNA (Bujalowski et al., 1986), the Tetrahymena ribozyme RNA (McConnell et al., 1997), and Bacillus subtilis P RNA (Beebe et al., 1996). Our previous results also showed that $\mathrm{Mg}^{2+}$ ions stabilized the conserved stem-loop structure (stem-loop IV) of RNA substrates for the LRV endoribonuclease and they are essential components for LRV capsid-dependent RNA cleavage (Ro and Patterson, 2000). Therefore, it is likely that the inhibitory effects of these antibiotics on LRV1-4 capsid endoribonuclease activity might be related to interfering with the proper folding of this structure, possibly by displacement of the $\mathrm{Mg}^{2+}$ ion(s). On the other hand, hygromycin $B$ is known to specifically block the translocation step on both $70 \mathrm{~S}$ and $80 \mathrm{~S}$ ribosomes (Cabanas et al., 1978), but it has not yet been reported whether it can directly inhibit RNase $P$, similar to neomycin and kanamycin. This study clearly demonstrated that the tested aminoglycosides inhibited the RNA cleavage activity in a dose response manner, but their magnitudes of inhibition varied greatly. Neomycin was a more potent inhibitor than kanamycin, while hygromycin B showed less inhibition than both aminoglycosides. These different magnitudes of inhibition by aminoglycosides on LRV14 capsid-driven endoribonuclease activity could be attributed to the number of amino groups ( 6 in neomycin, 4 in kanamycin, and 2 in hygromycin B) and the presence of an ammonium group at the R1 position of the aminoglycoside molecules that are important in the process of inhibition as suggested in RNase P (Mikkelsen et al., 1999; Tekos et al., 2000). Motif analysis of LRV1-4 capsid protein (EMBL/ GenBank U01899) using PROSITE database (Falquet et al., 2002) showed that the capsid protein did not bear any known RNase motifs. Also, EGTA-treated sucrose-purified LRV1-4 particles specifically lost the 
ability to generate the specific RNA cleavage product, due to disassembly of the viral capsid when EGTA is present (MacBeth and Patterson, 1995a), as many capsid proteins require $\mathrm{Ca}^{2+}$ for stable assembly (Brady et al., 1977). These facts lead us to check whether the virus assembly is closely related to the specific RNA cleavage activity. Previously, using the recombinant baculovirus expression system, several LRV1-4 capsid deletion mutants were constructed and their viral assembly was examined by sucrose gradient sedimentation and electron microscopy (EM) analysis (Cadd et al., 1994). Of these mutants, at least 24 amino acids of $\mathrm{N}$-terminus of capsid protein (N2 mutant) are required for the proper assembly, but 115 amino acids from $\mathrm{C}$-terminus of capsid protein (C1 mutant) are not necessary for the assembly (Cadd et al., 1994). For further delineating essential amino acid(s) for LRV1-4 assembly, we randomly chose four cysteine reidues important for protein primary structure by disulfide bond at positions 47 , 128,133 , and 194 from total 25 cysteine residues in LRV1-4 capsid protein (total 742 amino acids, EMBL/ GenBank U01899) and the cysteine residues were point-mutated to serine or arginine residues, not to alanine, based on single nucleotide sequence change for site-directed PCR mutagenesis. When the newlygenerating recombinant mutants, P1 (C47S), P2 (C128/ 133S), and P3 (C194R), were examined for their viral assembly ability by sucrose sedimentation analysis, only P3 mutant showed no proper virus assembly, similar to N2 mutant, suggesting that the 196 cysteine residue of LRV1-4 capsid protein is important for the viral assembly. Also, an $180-\mathrm{kDa}$ protein, previously depicted as dimer of capsid protein that was not dissociated under the denaturing gel conditions (Cadd and Patterson, 1994; Ro et al., 1997a), was not detected in the assembly-defective N2 and P3 mutants, suggesting that this tightly associated capsid form, not dissociated even under the denaturing gel condition used, is an essential component of the viral assembly. Interestingly, when in vitro RNA cleavage assay was performed with total eight recombinant LRV1-4 capsid proteins constructed previously (wild, C1, C2, N2, and N3) (Cadd et al., 1994) and newly (P1, P2, and P3) in this study, only the assemblydefective recombinant capsid proteins (N2 and $\mathrm{P} 3$ ) lost the specific RNA cleavage activity, similar to that the disassembled capsid protein by EGTA lost the cleavage activity (MacBeth and Patterson, 1995a). Additionally, neither LRV1-4 monomeric capsid protein purified from a polyacrylamide gel nor the insoluble and unassembled LRV1-4 capsid protein expressed in Escherichia coli and insect cells, respectively, exhibited the RNA cleavage activity (unpublished data). Taken together, these results lead us to believe that the site-specific cleavage at the nucleotide 320 is presumably mediated by the properly assembled LRV1-4 capsid proteins.

Despite many evidences that LRV1-4 capsid truly possesses the site-specific endoribonuclease activity on its own RNA transcript (MacBeth and Patterson, 1995a and b; MacBeth et al., 1997, Ro and Patterson, 2000, and in this study), however, it is unclear why the endoribonuclease activity is so low and the catalytic turnover is limited. The cleavage activity is truly dependent on the incubation time and the amount of enzyme added in the cleavage assay (Ro and Patterson, 2000), but we could not experience a complete cleavage of the RNA substrate we used. Actually, in LRV replication model (MacBeth and Patterson, 1998), the specific cleavage of LRV RNA genome by its own capsid protein has a potential risk for LRV propagation. If the endoribonuclease activity were unlimited, the LRV RNA genome would be mostly cleaved and LRVs could not maintain their persistent infection in host Leishmania cells. Therefore, the facts that only the fully assembled capsid proteins, not the capsid protein alone, possess the endoribonuclease activity can be one of the regulation mechanisms of LRV copy number in host cells. In fact, endoribonucleases are known to serve diverse roles in the generation of functional RNAs, the activation of expression from RNAs, and the physical and functional inactivation of RNAs (Belasco, 1993; Court, 1993). The finding that LRV viral capsid particles possess a site-specific endoribonuclease activity responsible for viral RNA genome cleavage is an intriguing component of Leishmaniavirus physiology, but direct evidences for the functional role of LRV capsid endoribonuclease is yet to be generated. Indirect evidences demonstrating that the $5^{\prime}$ end of LRV1 genomes is strongly conserved in both nucleotide sequence and RNA secondary structure (Scheffter et al., 1994), as well as data that support the functional role of the $5^{\prime}$ UTR of LRV as an internal ribosome entry site (IRES) for cap-independent translation in Leishmania (Maga et al., 1995), suggest that this $5^{\prime}$ UTR region is important for LRV replication. Also, data that Leishmania cellular proteins can bind specifically to cleave RNA of LRV1-4 produced by post-cleavage event (Saiz et al., 1999) support that the cleavage might alter the functionality of the viral transcript and regulate the viral propagation. The fact that only the properly assembled LRV1-4 virus particles possess the endoribonuclease activity shown in this study, implies that the cleavage event occurring after VLPs formation may be a mechanism regulating viral genome copy number for maintenance of a LRV persistence infection in Leishmania, along with recent discoveries that a Leishmania-encoded cysteine protease cleaves a Leishmaniavirus Gag-Pol polyprotein (Carrion et al., 2003) and 
a specific inhibition of the cysteine protease activity provides another regulatory mechanism for maintaining a low viral copy number in vivo (unpublished data).

Based on the data generated in this study for highlighting the enzymatic nature of LRV1-4 capsid protein, we conclude that the LRV capsid truly possesses an endoribonuclease function and the endoribonuclease activity may rely upon fully assembled virus particles for the specific cleavage of LRV1-4 viral RNA. Structural analysis of LRV1-4 wild and cleavage-deficient capsid proteins by cryo-electron microscopy (cryo-EM) study will be helpful for further delineating our suggestion.

\section{Acknowledgment}

This study was supported by a grant (R05-2000000-00102-0) from the Basic Research Program of the Korea Science \& Engineering Foundation and a NIH grant A128473 for MS, RC, and JLP.

\section{References}

von Ahsen U, Davies J, Schroeder R. Antibiotic inhibition of group I ribozyme function. Nature 1991;353:368-70

Armstrong TC, Patterson JL. Cultivation of Leishmania braziliensis in an economical serum-free medium containing human urine. J Parasitol 1994;80:1030-2

Bakker EP. Aminoglycoside and aminocyclitol antibiotics: hygromycin $B$ is an atypical bactericidal compound that exerts effects on cells of Escherichia coli characteristic for bacteriostatic aminocyclitols. J Gen Microbiol 1992;138:563-9

Beebe JA, Kurz JC, Fierke CA. Magnesium ions are required by Bacillus subtilis ribonuclease $P$ RNA for both binding and cleaving precursor tRNA ${ }^{\text {Asp }}$. Biochemistry 1996;35:10493505

Belasco JG. mRNA degradation in prokaryotic cells: an overview. In Control of messenger RNA stability (Belasco, J., and Brawerman, G., eds.), 1993, 3-12, Academic Press, San Diego, CA

Brady JN, Winston VD, Consigli RA. Dissociation of polyoma virus by the chelation of calcium ions found associated with purified virions. J Virol 1977;23:717-24

Bujalowski W, Graeser E, McLaughlin LW, Porschke D. Anticodon loop of tRNA ${ }^{\text {Phe. }}$ structure, dynamics, and $\mathrm{Mg}^{2+}$ binding. Biochemistry 1986;25:6365-71

Cabanas MJ, Vazquez D, Modolell J. Dual interference of hygromycin B with ribosomal translocation and with aminoacyl-tRNA recognition. Eur J Biochem 1978;87:21-7

Cadd TL, Keenan MC, Patterson JL. Detection of Leishmania RNA virus 1 proteins. J Virol 1993;67:5647-50

Cadd TL, MacBeth K, Furlong D, Patterson JL. Mutational analysis of the capsid protein of Leishmania RNA virus LRV1-4. J Virol 1994;68:7738-45
Cadd TL, Patterson JL. Synthesis of viruslike particles by expression of the putative capsid protein of Leishmania RNA virus in a recombinant baculovirus expression system. J Virol 1994;68:358-65

Carrion R. Jr, Ro YT, Patterson JL. Purification, identification, and biochemical characterization of a host-encoded cysteine protease that cleaves a Leishmaniavirus Gag-Pol polyprotein. J Virol 2003;77:10448-55

Chung IK, Armstrong TC, Patterson JL. Identification of a short viral transcript in Leishmania RNA virus-infected cells. Virology 1994;198:552-6

Court, D. RNA processing and degradation by RNase III. In Control of messenger RNA stability (Belasco $\mathrm{J}$ and Brawerman G, eds.), 1993, 71-116, Academic Press, San Diego, $\mathrm{CA}$

Eubank TD, Biswas R, Jovanovic M, Litovchick A, Lapidot A, Gopalan V. Inhibition of bacterial RNase P by aminoglycoside-arginine conjugates. FEBS Lett 2002;511:107-12

Falquet L, Pagni M, Bucher P, Hulo N, Sigrist CJ, Hofmann K, Bairoch A. The PROSITE database, its status in 2002. Nucleic Acids Res 2002;30:235-8

MacBeth KJ, Patterson JL. The short transcript of Leishmania RNA virus is generated by RNA cleavage. J Virol 1995a;69:458-3464

MacBeth KJ, Patterson JL. Single-site cleavage in the 5 '-untranslated region of Leishmaniavirus RNA is mediated by the viral capsid protein. Proc Natl Acad Sci USA 1995b; 92:8994-8

MacBeth KJ, Ro YT, Gehrke L, Patterson JL. Cleavage site mapping and substrate-specificity of Leishmaniavirus 2-1 capsid endoribonuclease activity. J Biochem 1997;122:193200

MacBeth KJ, Patterson JL. Overview of the Leishmaniavirus endoribonuclease and functions of other endoribonucleases affecting viral gene expression. J Exp Zool 1998;282:254-60

Maga JA, Widmer G, Lebowitz LL. Leishmania RNA virus 1 mediated cap-independent translation. Mol Cell Biol 1995; 15:193-200

McConnell TS, Herschlag D, Cech TR. Effects of divalent metal ions on individual steps of the Tetrahymena ribozyme reaction. Biochemistry 1997;36:8293-303

Mikkelsen NE, Brannvall M, Virtanen A, Kirsebom LA. Inhibition of RNase $P$ RNA cleavage by aminoglycosides. Proc Natl Acad Sci USA 1999;96:6155-60

Oh SH, Cho SY, Jin HS, Jeong DL, Jung SG, Oh KH. Expression and characterization of a mutant recombinant blood coagulation factor VIII ( $\mathrm{rFVIII}(\mathrm{m})$ ). Exp Mol Med 2002;34:233-8

Ro YT, Scheffter SM, Patterson JL. Specific in vitro cleavage of a Leishmania virus capsid-RNA-dependent RNA polymerase polyprotein by a host cysteine-like protease. J Virol 1997a;71:8983-90

Ro YT, Scheffter SM, Patterson JL. Hygromycin B resistance mediates elimination of Leishmania virus from persistently infected parasites. J Virol 1997b;71:8991-8 
Ro YT, Patterson JL. Identification of the minimal essential RNA sequences responsible for site-specific targeting of the Leishmania RNA virus 1-4 capsid endoribonuclease. J Virol 2000;74:130-8

Rosenstein SP, Been MD. Evidence that genomic and antigenomic RNA self-cleaving elements from hepatitis delta virus have similar secondary structures. Nucleic Acids Res 1991;19:5409-16

Saiz M, Ro YT, Wirth DF, Patterson JL. Host cell proteins bind specifically to the capsid-cleaved $5^{\prime}$ end of Leishmaniavirus RNA. J Biochem 1999;126:538-44

Scheffter S, Widmer G, Patterson JL. Complete sequence of Leishmania RNA virus $1-4$ and identification of conserved sequences. Virology 1994;199:479-83

Scheffter SM, Ro YT, Chung IK, Patterson JL. The complete sequence of Leishmania RNA virus 2-1, a virus of an Old World parasite strain. Virology 1995;212:84-90

Spahn CM, Prescott CD. Throwing a spanner in the works: antibiotics and the translation apparatus. J Mol Med 1996; 74:423-39

Stuart KD, Weeks R, Guilbride L, Myler PJ. Molecular organization of Leishmania RNA virus 1. Proc Natl Acad Sci USA 1992;89:8596-600

Sun W, Jun E, Nicholson AW. Intrinsic double-stranded-RNA processing activity of Escherichia coli ribonuclease III lacking the dsRNA-binding domain. Biochemistry 2001;40:14976-84

Tarr PI, Aline RF, Smiley BL, Keithly J, Stuart K. LR1: a candidate RNA virus of Leishmania. Proc Natl Acad Sci USA 1988;85:9572-5

Tekos A, Tsagla A, Stathopoulos C, Drainas D. Inhibition of eukaryotic ribonuclease $P$ activity by aminoglycosides: kinetic studies. FEBS Lett 2000;485:71-5

Widmer G, Comeau AM, Furlong DB, Wirth DF, Patterson $\mathrm{JL}$. Characterization of a RNA virus from the parasite Leishmania. Proc Natl Acad Sci USA 1989;86:5979-82 